\title{
Lenalidomide for bortezomib-resistant multiple myeloma
}

Chiara Briani, Tamara Berno, Marta Campagnolo and Renato Zambello

We read with great interest the paper by Ludwig and Zojer reporting a patient with bortezomib-resistant multiple myeloma who achieved renal recovery after lenalidomide therapy (Renal recovery with lenalidomide in a patient with bortezomib-resistant multiple myeloma. Nat. Rev. Clin. Oncol. 7, 289-294; 2010). ${ }^{1}$

A similar case was reported in 2009 by Layzer and Wolf in a patient with multiple myeloma and refractory polyneuropathy whose condition significantly improved after treatment with lenalidomide. ${ }^{2}$ The reduced neurotoxic effect of lenalidomide ${ }^{3}$ compared with thalidomide ${ }^{4}$ is well known, and results from a retrospective study reported that six of nine patients with bortezomib-induced neuropathy had symptomatic improvement after therapy with lenalidomide. ${ }^{5}$ In line with these encouraging data, we would like to share our group's preliminary experience with lenalidomide in patients with chemotherapy-induced peripheral neuropathy (CIPN).

In a single-center, prospective study we are evaluating the clinical course of CIPN in patients previously treated with bortezomib and/or thalidomide who are currently receiving therapy with lenalidomide ( $25 \mathrm{mg}$ per day for a 21-day cycle) for relapsed and/or refractory multiple myeloma. The control group comprises patients with CIPN whose multiple myeloma is still in remission. Patients are examined with the Total Neuropathy Score clinical version (TNSc), previously validated against the most common neurotoxicity scales
(National Cancer Institute-Common Toxicity Scale 2.0, Eastern Cooperative Oncology Group [ECOG] performance status); the TNSc is able to detect presence, severity, and longitudinal changes in CIPN. ${ }^{6}$ TNSc $>2$ is considered significant for CIPN. Patients without CIPN at baseline were not excluded from this study. Pain was assessed with the Numeric Rating Scale (NRS). At enrollment 25 of the 40 patients presented symptoms and signs of CIPN (mean TNSc 5.6, median 5, range 3-15), which was confirmed in half of the patients by neurophysiological studies. Of the 40 patients currently receiving lenalidomide therapy, 19 (mean age 66 years \pm 8 years) were available for evaluation with at least 6 months of follow-up, 12 of whom were also available at 1 year. After 6 months of follow-up, hematological response was documented in 17 of 19 patients, whereas two patients were in disease progression. Neurological evaluation revealed improvement (seven patients) or stability (11 patients) of symptoms and improvement of TNSc after 6 months (mean TNSc improvement 3.6, median 3, range 2-8), that persisted unchanged in 11 of 12 patients at 1 year. The only patient whose TNSc had worsened at 6 months had an improvement to baseline value after 1 year. The improvement of symptoms was more evident in patients with high baseline TNSc. Patients with TNSc 0 at baseline (that is, with no CIPN), remained unchanged after 6 months and 12 months of lenalidomide therapy. NRS and ECOG performance status did not change during lenalidomide therapy.

In conclusion, using validated clinical and instrumental analyses, the preliminary results of our prospective study suggest that during lenalidomide therapy CIPN seems to improve, regardless of multiple myeloma response. The results of the ongoing follow-up study on a wider population of patients will help confirm these data.

Department of Neurosciences (C. Briani, M. Campagnolo), Department of Clinical and Experimental Medicine (T. Berno,

R. Zambello), University of Padova, Via

Giustiniani, Padova, Italy.

Correspondence to: C. Briani chiara.briani@unipd.it

doi:10.1038/nrclinonc.2010.31-c1

\section{Acknowledgments}

The authors thank Dalla Torre Chiara, Candiotto Laura and Lucchetta Marta for their precious contribution to the study, and Perlotto Novenia for the neurophysiological studies.

Competing interests

The authors declare no competing interests.

1. Ludwig, H. \& Zojer, N. Renal recovery with lenalidomide in a patient with bortezomibresistant multiple myeloma. Nat. Rev. Clin. Oncol. 7, 289-294 (2010).

2. Layzer, R. \& Wolf, J. Myeloma-associated polyneuropathy responding to lenalidomide. Neurology 73, 812 (2009).

3. Galustian, C. \& Dalgleish, A. Lenalidomide: a novel anticancer drug with multiple modalities. Expert Opin. Pharmacother. 10, 125-133 (2009).

4. Briani, C. et al. Thalidomide neurotoxicity: prospective study in patients with lupus erythematosus. Neurology 62, 2288-2290 (2004).

5. Badros, A. et al. Neurotoxicity of bortezomib therapy in multiple myeloma: a single-center experience and review of the literature. Cancer 110, 1042-1049 (2007).

6. Cavaletti, G. et al. The Total Neuropathy Score as an assessment tool for grading the course of chemotherapy-induced peripheral neurotoxicity: comparison with the National Cancer Institute-Common Toxicity Scale. J. Peripher. Nerv. Syst. 12, 210-215 (2007). 